\title{
USE OF BETEL LEAVES IN PEDIATRIC STOMA CARE
}

\author{
MAHUQ ${ }^{1}$, AKMM RAHMAN ${ }^{2}$, THOSSAIN ${ }^{3}$
}

\begin{abstract}
:
Aim of the study: To find out a practicable, cost effective and easily available alternative of commercial stoma care devices for pediatric patients in a developing country.

Methods: The study was conducted in the department of Pediatric Surgery in Dhaka Medical College Hospital, Bangladesh, during two years period from May 2009 to April 2011. Number of patients was 162 with age range 2 days to 7 years, mean age 2.3 years. As early as a colostomy or ileostomy had started functioning, a central hole was made in a betel leaf. The hole could just snugly accommodate the stoma. After applying a layer of zinc oxide paste over the peristomal skin the leaf was placed as such the stoma protrudes through the hole. The smooth shiny surface of the leaf would face upwards and rough surface downwards. Another intact betel leaf with the shiny surface also facing upwards would cover the stoma. The leaves did not act as a reservoir rather simply acted as a barrier between the effluent and the peristomal skin. Fifty seven patients with colostomy and 11 with ileostomy used stoma appliances (wafer with bag). Eighty six patients with colostomy and 8 patients with ileostomy used betel leaves and zinc oxide paste. Mean length of time between creation and closure of a colostomy was 9 months and that of an ileostomy was three months. It was ensured that each individual patient had used his or her respective stoma care method till closure of the stomas. Two parameters were used to evaluate the outcome of the above mentioned stoma care methods: (a) peristomal skin excoriation and (b) insult
\end{abstract}

1. Dr. Md. Ashraf UI Huq, Assoc. Prof., Dept of Pediatric surgery, Dhaka Medical College \& Hospital, Bangladesh.

2. Dr. A K M Mizanur Rahman, Registrar, Dept. of Pediatric Surgery, Dhaka Medical College \& Hospital, Bangladesh.

3. Dr. Tahmina Hossain, Asst. Prof., Dept. of Pediatric Surgery, Dhaka Medical College \& Hospital, Bangladesh.

Correspondence: Dr. Md. Ashraf UI Huq, MS, FICS, PhD, Associate Professor, Dept of Pediatric surgery, Dhaka Medical College \& Hospital, Dhaka, Bangladesh. Email address: kazal64@yahoo.com to the stomal mucosa in the form of ulceration. Result: Among the patients who used stoma appliances $21.53 \%$ developed peristomal skin excoriation, $7.69 \%$ developed mucosal ulceration and $6.34 \%$ patients developed local hypersensitivity reaction to stoma adhesive. On the contrary, patients who were managed with betel leaves $20.93 \%$ developed peristomal skin excoriation and $8.13 \%$ developed mucosal ulceration. No incidence of allergic reaction to local application of betel leaf and zinc oxide occurred. Average cost for betel leaves was less than 0.50 US $\$$ per month in comparison to about 30 US\$ for ostomy appliances. Conclusion: Betel leaves may be used as a cheap, easily available, non irritant and effective alternative of commercial appliances to protect the stomas and peristomal skin in pediatric patients.

Key words: Stoma care, betel leaf.

\section{Introduction:}

History of colostomies date back to $18^{\text {th }}$ century, ileostomies evolved later. ${ }^{4}$ Despite sporadic early successes, surgeons were reluctant, even strongly opposed to perform these drastic procedures, which were associated with major complications. ${ }^{5}$ Temporary colostomy and ileostomy are common life saving procedures in neonates with intestinal obstruction and often are adjunct of multistage gastrointestinal reconstructive surgery in children. Vesicostomy, colpostomy and esophagostomy are also not very uncommon. Appliance like a stoma bag is usually advised to collect the expelled out effluent and to prevent contact of it with the peristomal skin. Long term contact of fecal matter with abdominal skin causes irritation, dermatitis and excoriation of the area around the stoma. Damage is even more severe in case of ileostomy because the effluent coming out through an ileostomy is rich of digestive enzymes. 


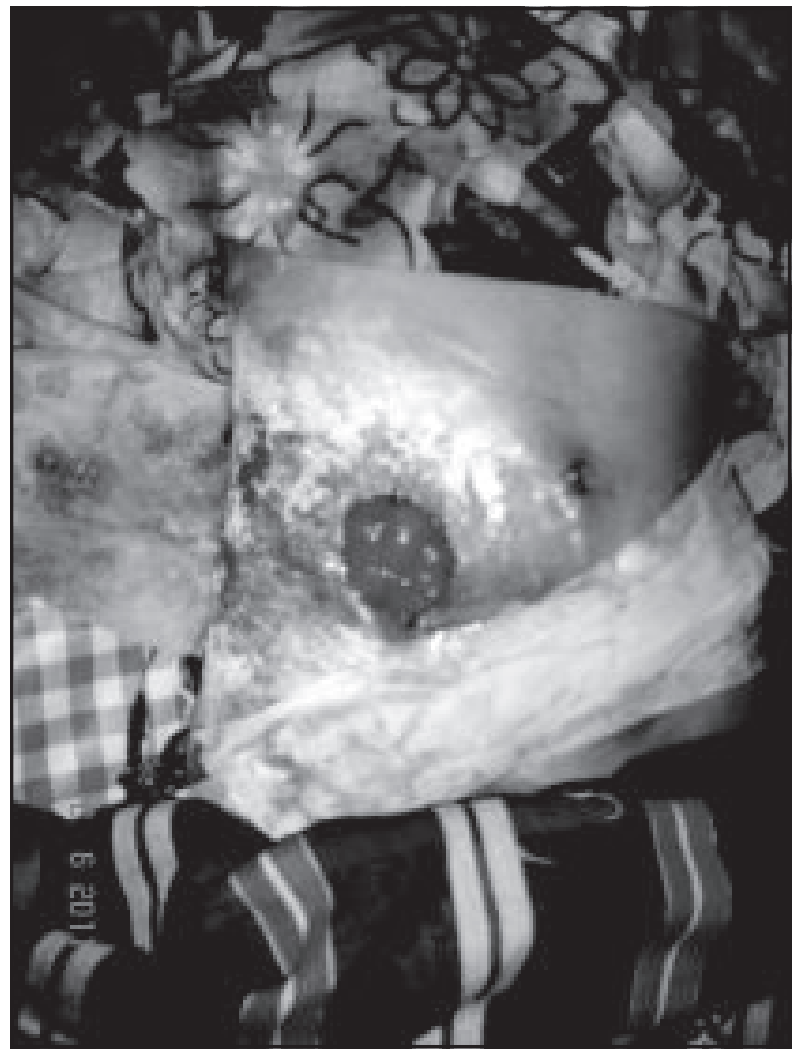

Fig.1: Peristomal skin excoriation

A well fitted wafer with a stoma bag of appropriate size can prevent or reduce effluent induced skin damage. Stoma appliances for neonates, small children and even for the older ones are, in practice, not available in most of the medical retailers in Bangladesh. It is also beyond the capacity of most of the parents to carry the expenses of one or two sets of stoma appliances every week. Never the less this is also difficult to keep a stoma appliance in position for a reasonable period in children especially the younger ones. Another important fact is that, regular irrigation through the distal stoma of a loop colostomy is essential to prevent fecaloma formation in the rectosigmoid. It is more important in cases like anorectal malformation, Hirschsprung's disease and meconium ileus. A wafer based collecting bag usually difficult to fit in neonates and small children for lack of adequate space in the abdominal wall. Rather, a single piece stoma bag with adhesive is directly applied over their stomas. In such cases distal irrigation is not possible keeping the stoma bag in situ. It is definitely a huge economic burden on the parents to parchage one or two bags daily.

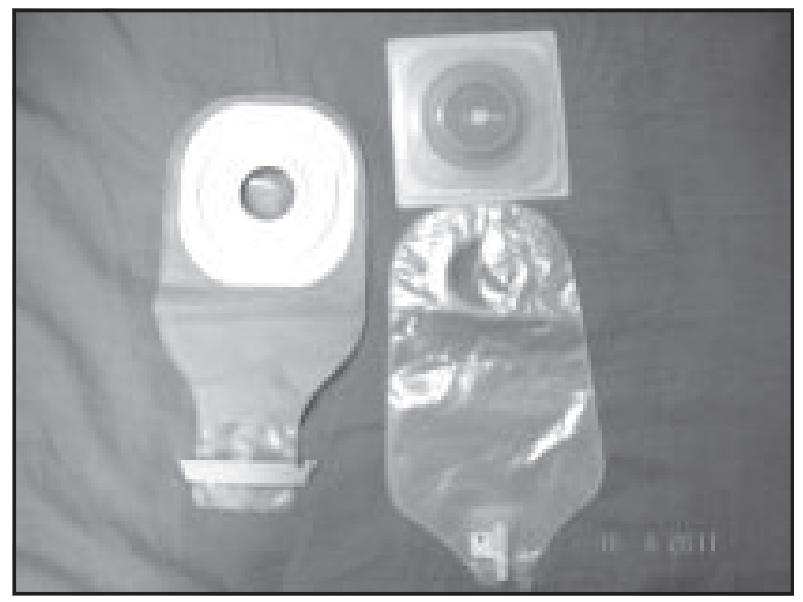

Fig. 2: Stoma appliances

A set of two betel leafs (Piper betle), one with a central hole equal to the stoma size, and the other placed over the exposed gut mucosa serves two purposes: (a) It, as a mechanical barrier, prevents direct contact of effluent with the peristomal skin and (b) the other leaf prevents contact and friction of the stomal mucosa with patients' clothing. A betel leaf has a smooth surface which has an apparent soothing effect on skin and delicate mucosa. The chemical or pharmacological component(s) of the betel leaf that produce the job is yet to be discovered. Distal irrigation is easy removing the leaf over the stoma.

\section{Materials and method:}

Study design: This was a prospective, comparative and noninvasive study done in a two year period from May 2009 to April 2011.

Study place: The study was conducted in the department of Pediatric Surgery in Dhaka Medical College Hospital, Bangladesh.

Target group and sample size: All pediatric patients with a colostomy or ileostomy were included in the study. Total number of patients was 162 with age range 2 days to 7 years; mean age $14 \pm 2$ months. Patients were initially divided into two groups. Group $A$ included the patients with colostomy $(n=143)$ and group $B$ with ileostomy $(n=19)$. Each group again subdivided into two subgroups: group1 used betel leaves and group2 used commercially available appliances (wafer with bag) for care of the stoma and its surrounding skin. Out of 143 colostomy patients $86(60.13 \%)$ used betel leaves and zinc oxide paste $\left(A_{1}\right)$ and the other 57 $(39.87 \%)$ used stoma appliances $\left(A_{2}\right)$. Of 19 ileostomy patients $11(57.89 \%)$ used betel leaf and zinc oxide $\left(B_{1}\right)$ and the rest $8(42.11 \%)$ patients used stoma appliances $\left(\mathrm{B}_{2}\right)$. 


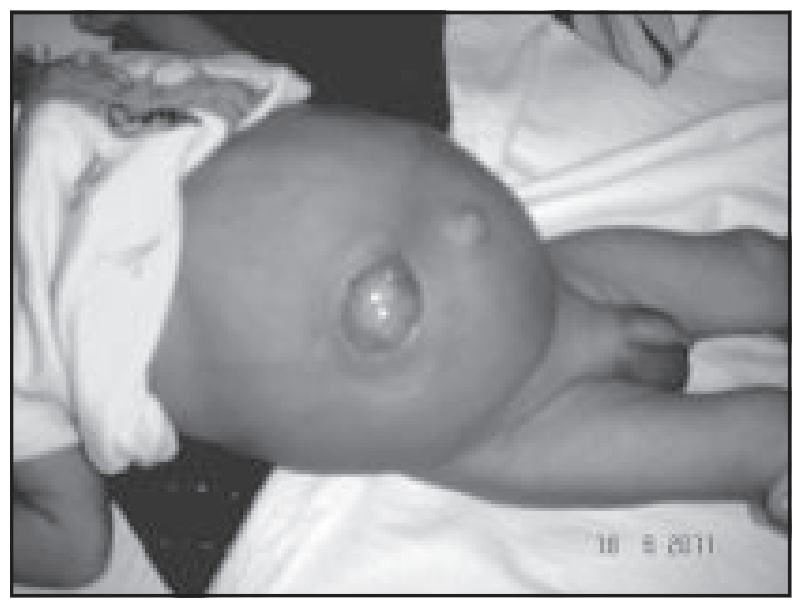

Fig. 3: Transverse loop colostomy in a Patient of Hirschsprung's disease

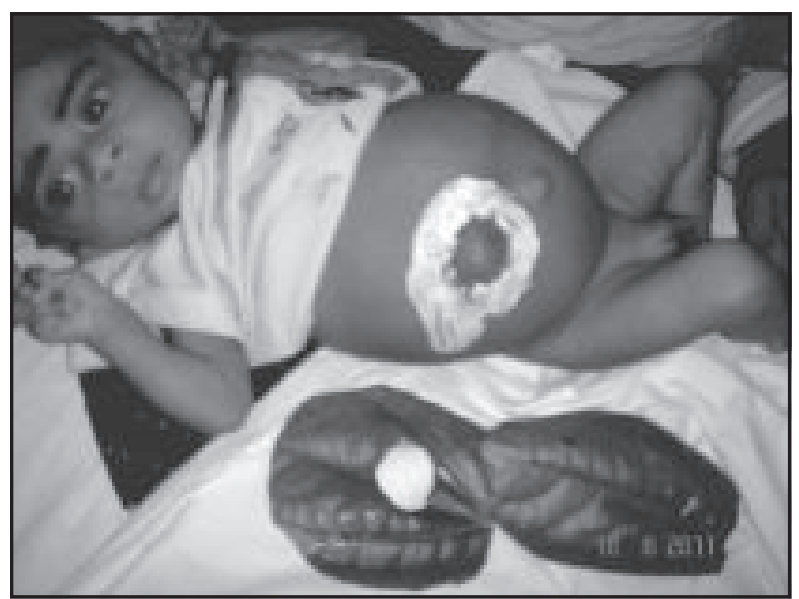

Fig. 4a: Step 1: Application of zinc oxide paste and sizing the betel leaves

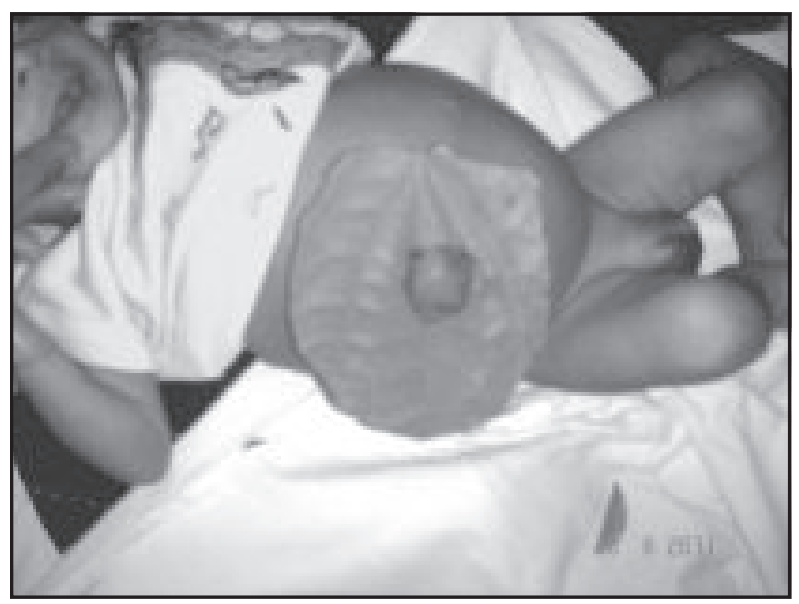

Fig. 4b Step 2: Fitting the leaf with central hole around the stomas

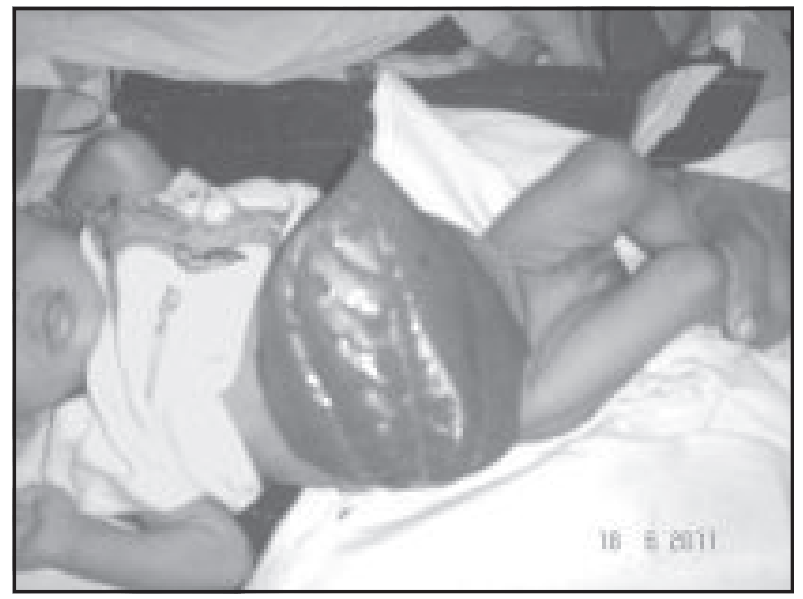

Fig. 4c Step 3: Placing the other leaf over the stomas

Sample collection: Patients admitted in the department of Pediatric Surgery in Dhaka Medical College Hospital, Bangladesh who underwent colostomy or ileostomy for different surgical causes were included in the study. These diseases included anorectal malformations, Hirschsprung's disease, intestinal atresia, enterocolitis, intussusception, enteric fever and ascariasis. All the colostomies and ileostomies were temporary. Mean length of time between colostomy and its closure was 8 months and that of ileostomy closure was 3 months. Choice of betel leaf or stoma bag was dependent on their parents' economic solvency and availability of the device.

Inclusion criteria: Patients with age below 12 years having temporary colostomy or ileostomy were included in the study.

Exclusion criteria: Patients more than 12 years and patients with permanent colostomy or ileostomy were excluded in the study.

Parameters: Evaluation of the use of the study instruments was based on two parameters: (a) peristomal skin excoriation and (b) insult to the stomal mucosa in the form of ulceration.

Procedure: Use of commercially available colostomy or ileostomy appliance is as usual - an adhesive paste (Stomahesive) is applied on the peristomal skin. Over this a wafer is anchored after enlarging the central opening according the size of the stoma. Then a collecting bag is bag attached to the wafer. The outlet of the bag is clipped so that the effluent can be collected and drained time to time. This type of 
appliance can be kept in situ for a few days. Sometimes a disposable bag is directly attached to the skin without a wafer. When a wafer or a bag is loosened the old glue is wiped out and a new layer is applied.

Parents who are unable to buy or unwilling to use a stoma bag are advised to use betel leaves to protect the stoma and its adjoining skin. In that case initially zinc oxide paste is applied in the skin around the stoma. As soon as a colostomy or ileostomy has started functioning, a central hole is made in a betel leaf. The hole can just snugly accommodate the stoma. Then the leaf is placed on the stoma so that it protrudes through the hole. The smooth shiny surface of the leaf faces upwards and rough surface downwards. Another intact betel leaf with the shiny surface facing upwards covers the stoma. The leaves serve as a barrier between the effluent and the peristomal skin. The effluent is washed away from the leaves after each bowel movement. The leaves can usually be reused after cleaning.

\section{Result:}

Assessment of the stomas and the adjacent skin were done on monthly interval. A checklist was used for individual patient. Information in the checklist included maceration, bleeding, granulation and ulceration of exposed mucosa of the stomas, as well as excoriation, ulceration, fungal and bacterial infection and irritant or allergicand contact dermatitis of the peristomal skin. However two parameters have been used to evaluate the outcome of the two methods of stoma care. The parameters are (a) peristomal skin excoriation and (b) insult to the stomal mucosa in the form of ulceration.

Out of $65(57+8)$ patients who used ostomy bags 14 $(21.53 \%)$ developed peristomal skin excoriation and 5 (7.69\%) developed mucosal ulceration. 8 (6.34\%) patients developed local hypersensitivity reaction to stoma adhesive. On the contrary, out of 86 patients who were managed with betel leaf $18(20.93 \%)$ developed peristomal skin excoriation and 7 (8.13\%) developed mucosal ulceration. No patient developed allergic reaction to local application of betel leaf. It was estimated that monthly costs for betel leaves were 10 to 15 cents, whereas ostomy appliance cost was about 30US\$.

\section{Discussion:}

An enterostoma in a child is a major disruption of normality and frequently leads to substantial psychological trauma for the child and the parents. However, most of the stomas in pediatric age group are temporary, and correction of the underlying problem very often leads to closure of the diverting opening. ${ }^{1}$ With the exception of feeding access, more than one half of the stomas are placed in the neonatal period and another one fourth in children younger than one year of age. ${ }^{2}$ Peristomal skin complications are the most common reason for ostomy patients visit an outpatient service. Prevention and management of peristomal skin complications are critical components of ostomy care. ${ }^{3}$ Identifying risk factors for the occurrence of peristomal skin complications and the clinical features can help optimum assessment and management approaches. Analysis of medical records of patients who underwent colostomy reveals two types of complications: stomal and peristomal. Stomal complications include maceration, bleeding, ulceration, necrosis, prolapse, retraction or stenosis of the stoma. Peristomal complications include irritant or allergic contact dermatitis, folliculitis, mechanical damage, hyperplastic granulation, bacterial and candidal infection and parastomal hernia. Irritant contact dermatitis, occurring in $15.5 \%$, is the most common peristomal complication. ${ }^{4}$

Treatment can be based on etiology such as chemical injury (irritant contact dermatitis, pseudo verrucous lesions, and encrustations); mechanical injury (pressure/shear, stripping, mucocutaneous separation, mucosal transplantation); infection (Candidiasis, folliculitis); immunologic disorders (allergic contact dermatitis); and disease-related lesions (varices, pyoderma gangrenosum etc.). The importance of prevention and the impact of having access to knowledgeable care providers cannot be over-emphasized. ${ }^{2}$

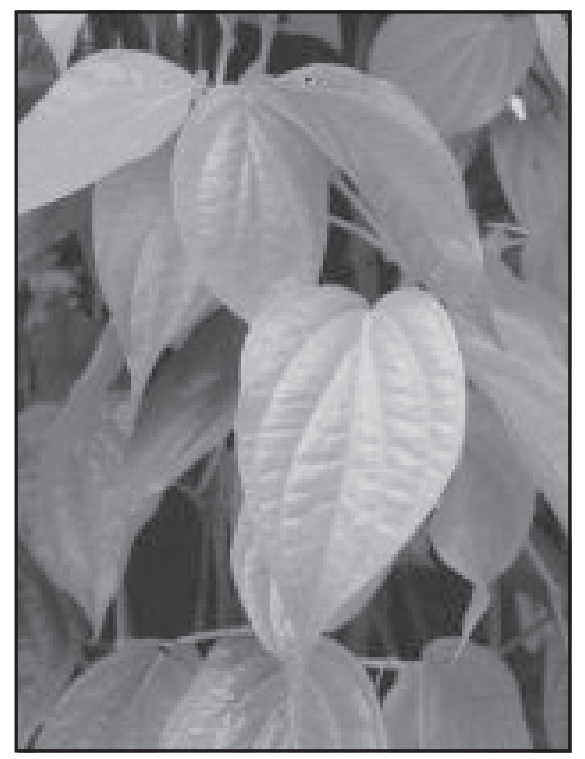

Fig: 5: Betel leaves in a vine 
The betel plant is a slender aromatic creeper having heart shaped, smooth, shining and long stalked leaves with pointed apex. Scientific name of the plant is Piper betle. Betel morsel (betel nut and other ingredients like quick lime, cardamom etc. folded in betel leaf) or pan supari is extensively taken in south Asia. Betel leaf is little sour in taste but is a popular mouth freshener which is usually used as a masticatory substance. It is a digestive, carminative, anti flatulent and stimulant. ${ }^{1}$ The usage of betel leaf in various medical and surgical conditions is cited as far back as two thousand years. The leaf consists of $84.5 \%$ water, $3.1 \%$ protein, $0.8 \%$ fat, $2.3 \%$ minerals, $2.3 \%$ fibers and $6.1 \%$ carbohydrate. Its mineral and vitamin contents are calcium, carotene, riboflavin, niacin, thiamin and vitamin $\mathrm{C}^{2}$

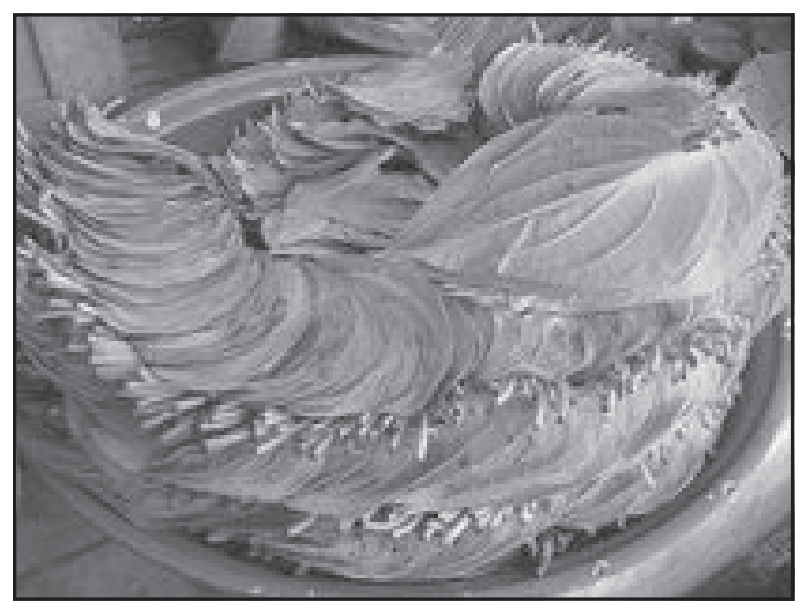

Fig: 6: Betel leaves in market

It also contains a powerful antiseptic phenol named chavicol. Local application of this leaf helps remedy of boils, sore throat, arthritis and orchitis. It also helps wound healing when extracted betel leaf juice is applied on the wound. There are some reported systemic functions of betel leaf, like, dieresis, relieving neurological pain etc. These factors encouraged us to use this leaf in care of abdominal stomas. Easy availability and low price of betel leaf is also an inciting factor.

In fact we could not identify the specific component or components which appear to be responsible for the beneficiary role of betel leaf in stoma care. To be frank the concept of using this leaf in stoma care is still in a very primary stage. More extensive and multi centered use of betel leaf in stoma care as well as intensive biochemical analysis of this leaf should be emphasized.

\section{Conclusion:}

No doubt, a commercial stoma appliance in the form of a well fitted stoma bag is best for better care of a colostomy or ileostomy and the adjecent skin. But in an underdeveloped country like Bangladesh, it is often beyond economic capability of parents to purchase stoma appliances regularly on a long term basis. It is also not easily available in the small towns and rural areas of the country. In such a situation, betel leaves may be used as a cheap, easily available, nonirritant and effective alternative of appliances to care a stoma and peristomal skin in pediatric patients.

\section{Acknowledgement:}

We would like to acknowledge Prof. Tahmina Banu of Chittagong Medical College \& Hospital, who was the first to use Betel Leaf for stomal care in children in Bangladesh.

\section{References}

1. Michael WLG, Cataldo PA, 'Intestinal stomas: Principles, techniques and management', St. Louis, Quality Medical Publishing, 1993.

2. Scharli WF, 'The history of colostomy in childhood', Prog Pediatr Surg, 1986; 20:188-198.

3. Wu H \& Wiener $\mathrm{E}$, 'Stomas in small and large intestine', In: Grosfeld JL, O'Neill Jr. JA, Fonkalsrud EW \& Coran AG (eds), Pediatric Surgery. 6th edn, vol. 1, Philadelphia: Mosby; 2006; 1:1479 - 1490.

4. Millar AJ, Lakhoo K, Rode M, et al, 'Bowel stomas in infants and children: A five yea audit of 203 patients', S Afr J Surg, 1993; 31:110-113.

5. Rolstad BS, Erwin-Toth PL, 'Peristomal skin complications: prevention and management', Ostomy Wound Manage, 2004; 50(9):68-77.

6. Irina K, Sergey A, Svetlana F and Genadiy V, 'The development and use of algorithms for diagnosing and choosing treatment of ostomy complications: Results of a prospective evaluation,' Ostomy wound management, 2011; 57(1): 20-27.

7. Paan-Betel leaf: Green leaf that cures, February 2009; available at webmaster@infotech.co.id.

8. 'Origin, distribution and composition of Betel Leaf', Herbs Guide, March 2009; available at http// www.online/herbs-guide.htm.

9. Paan-Betel leaf: Green leaf that cures, February 2009; available at webmaster@infotech.co.id.

10 'Origin, distribution and composition of Betel Leaf', Herbs Guide, March 2009; available at http// www.online/herbs-guide.htm. 